\title{
Construção de um Laboratório de Lógica para Programação dedicado a Cursos de Graduação
}

\author{
Laura Quevedo Jurgina ${ }^{1}$, Andressa Werner Krause Pereira1, Ana Amélia \\ Traversi $^{1}$, Gabriel Rocha Silveira ${ }^{1}$, Raquel de Souza Zimmer ${ }^{1}$, Placida Giuliane \\ Silveira De Oliveira ${ }^{1}$, Darlei Matheus Schmegel ${ }^{1}$, Dauan Ghisleni Zolinger ${ }^{1}$, \\ Moniele K. Santos ${ }^{1}$, Gerônimo G. Z. Lemos ${ }^{1}$, Bruno C. Alves ${ }^{1}$, Heitor F. M. dos \\ Santos $^{1}$, Gerson L. de Menezes ${ }^{1}$, Leomar Soares da Rosa Júnior ${ }^{1}$ \\ ${ }^{1}$ Centro de Desenvolvimento Tecnológico \\ Universidade Federal de Pelotas (UFPel) - Pelotas - RS - Brasil \\ \{lqjurgina, awkpereira, aatraversi, grsilveira, rdszimmer, \\ pgsdoliveira, dmschmegel, dgzolinger, mksantos, ggzlemos, bcalves, \\ hfmdsantos, gldmenezes, leomarjr\}@inf.ufpel.edu.br
}

\begin{abstract}
This work describes a workshop planned to meet the needs identified in undergraduate students during their course of programming. Logic is a fundamental concept that must be exercised and implemented in order to transform programming into simpler and better-understood activity. In addition to semantics and syntax, logical thinking is necessary. In this context, a logic workshop was proposed to assist students from different undergraduate courses at UFPel, and all regular participants obtained satisfactory results.
\end{abstract}

Resumo. Este trabalho descreve uma oficina planejada para suprir as necessidades identificadas nos alunos de graduação na disciplina de introdução à programação. A lógica é um conceito fundamental que deve ser exercitada e implementada para que programar seja uma atividade mais simples e melhor compreendida. Além da semântica e da sintaxe é necessário pensamento lógico. Uma oficina de lógica foi desenvolvida para auxiliar estes alunos dos diversos cursos de graduação da UFPel, e todos os participantes assíduos obtiveram resultados satisfatórios.

\section{Introdução}

A lógica é essencial para o aprendizado de programação, o seu principal objetivo é demonstrar técnicas capazes de solucionar problemas. É necessário um raciocínio para construir o passo a passo utilizando os procedimentos que solucionarão uma tarefa. $\mathrm{Na}$ lógica de programação, aprende-se a colocar as instruções - de um programa - em uma sequência lógica, e isso é fundamental para o correto funcionamento do mesmo. Além disso, ela auxilia o desenvolvimento da sagacidade que é primordial no desenvolvimento de programas. Quando o aluno não possui as habilidades essenciais para tal, encontram dificuldades, que podem culminar na sua reprovação ou evasão [Barbosa 2011].

Segundo França et. al. (2013) o fundamento de computação como ciência, é restrito e contempla apenas estudantes que escolhem cursos superiores e técnicos de áreas semelhantes. Por outro lado, Tucker (2006) discute a importância de que em pleno 
século XXI, cada cidadão deva ter conhecimento de pelo menos os princípios básicos da Ciência da Computação.

Os cursos de Ciência da Computação e Engenharia de Computação, na Universidade Federal de Pelotas (UFPel), possuem disciplinas que ensinam lógica, antes de ensinarem programação de computadores. Porém, os demais cursos da UFPel que possuem disciplinas de programação de computadores não possuem em seus currículos a disciplina de lógica - a base para que o aluno consiga entender plenamente programação. Visto essa deficiência no currículo, o aluno começa a cursar a disciplina e esbarra na dificuldade para compreender o conteúdo abordado em aula.

O grupo PET-Computação, analisando essas questões, desenvolveu um projeto de lógica de programação, com o objetivo de ensinar a lógica para alunos não pertencentes a área da computação. Para sua construção, foi realizada uma pesquisa online para verificar o interesse da comunidade em participar da atividade. A resposta foi positiva evidenciando a necessidade desta ação. O Projeto laboratório de lógica para programação foi motivado por duas razões: levar conhecimento sobre programação básica e lógica de programação à estudantes de fora da área da computação que possuam interesse pessoal no assunto e auxiliar os alunos que tem cadeira de programação de computadores a compreenderem a lógica de programação.

\section{Trabalhos Relacionados}

Os autores Pereira et. al. (2019) relatam a realização de uma capacitação para professores do Ensino Básico, com o objetivo de desenvolver um aplicativo para dispositivo móvel. Para o estudo foi utilizada a ferramenta plataforma App Inventor que permite a programação sem conhecer conceitos de lógica, porém ao decorrer do experimento foram exercitados a lógica de programação e programação baseada em blocos. Como resultado, diversos aplicativos diferentes foram desenvolvidos, mesmo com a dificuldade inicial de aprendizado de uma nova tecnologia, o ensino de lógica despertou o desejo de seguir programando e explorando este universo.

Na revisão sistemática apresentada por [Watson e Li 2014] foi encontrada uma taxa de 32,3\% de alunos que são reprovados em disciplinas introdutórias de programação. Buscando nova práticas de ensino e abordagens, [Francisco, Pereira Júnior e Ambrósio 2017] realizaram também uma revisão sistemática para identificar as novas ferramentas empregadas para mitigar este índice. $\mathrm{O}$ foco deste estudo foi verificar o grau de dificuldade dos problemas empregados nos exercícios de programação. $\mathrm{O}$ trabalho conclui que o aluno precisa conhecer a linguagem de computação, sua semântica e sintaxe, todavia também é necessário compreender que existe multidisciplinaridade quando se pensa em programar.

Existe a demanda do ensino de lógica de programação desde o Ensino Básico, para que os educandos construam o conhecimento necessário para utilizar a programação no seu cotidiano. O Scratch é uma linguagem gráfica de programação, desenvolvida no Massachusetts Institute of Technology (MIT) e possui como objetivo ensinar programação de maneira lúdica e intuitiva para crianças a partir de 8 anos de idade. [De Oliveira at. al. 2014] discutem um relato sobre a implementação de um curso, utilizando Scratch para alunos do $9^{\circ}$ ano do Ensino Fundamental, onde concluem que a atividade foi satisfatória, pois as crianças envolvidas conseguiram atingir os objetivos propostos e adquiriram conhecimento sobre a área de computação. Os autores 
também ratificam a necessidade da reprodução dessas atividades no ensino público como meio de auxilio no processo de ensino-aprendizagem, pois assim os alunos terminam o Ensino Básico mais críticos e confiantes.

Segundo Bosse e Gerosa (2015), a disciplina de introdução à programação está presente em diversos cursos nas áreas de exatas, humanas e biológicas. Durante o curso, os alunos enfrentam grandes dificuldades em compreender os conceitos apresentados que podem ocasionar em reprovações, trancamentos e evasões. Todavia, não são realizados estudos que quantificam estes percentuais. Os autores realizaram o levantamento dos dados, ao total 19.859 matrículas, e analisaram o volume de reprovações nos últimos cinco anos na Universidade de São Paulo (USP). O percentual médio de reprovações/trancamentos é de $29,31 \%$ e cerca de $25 \%$ dos estudantes aprovados cursaram a disciplina mais de uma vez.

\section{Metodologia}

A construção da oficina de lógica contou com três fases: pesquisa online; seleção do material e planejamento das aulas e execução dos encontros.

$\mathrm{Na}$ primeira fase, uma pesquisa on-line foi realizada para avaliar o interesse dos alunos por um projeto que discutisse os conceitos de lógica de programação. Para tal, um formulário foi disponibilizado para coletar o curso de graduação e período do aluno interessado e, também, os horários e campus da Universidade em que ele teria disponibilidade de frequentar os encontros, para que a oficina fosse acessível ao maior número de interessados.

O formulário foi amplamente divulgado pelas redes sociais, e principalmente em grupos envolvendo a Universidade Federal de Pelotas (UFPel) no Facebook. Dessa forma, muitos estudantes foram alcançados. Durante uma semana, foi possível notar grandes resultados e procura por parte dos discentes. A partir das respostas, selecionouse os alunos para participarem do projeto; foi dado prioridade ao local e ao horário mais solicitado.

Os encontros foram planejados buscando construir conceitos com base nos tópicos julgados essenciais para a plena compreensão da disciplina programação de computadores. Para construir o material didático para a realização da oficina, foi utilizado como principal referência teórica de conhecimento o livro C Total e Completo [Schildt 1996]. Esse livro foi utilizado, pois apresenta vários exemplos que aplicam a lógica de programação na própria linguagem $\mathrm{C}$, evidenciando a necessidade da lógica na programação. A relação entre os temas discutidos e os encontros pode ser visualizado na Tabela 1.

A realização da oficina contou com oito encontros que foram ministrados em oito semanas, ou seja, uma aula por semana, com duração de $1 \mathrm{~h} 30 \mathrm{~min}$, totalizando $12 \mathrm{~h}$. Cada reunião abordou um assunto diferente e importante, seguindo uma ordem cronológica, ou seja, os conceitos eram acumulativos, sendo necessária a presença em todos os dias para obter o conhecimento completo do assunto.

O planejamento do encontro foi organizado da seguinte maneira: os 10 minutos iniciais seriam dedicados a uma revisão rápida da aula anterior - exceto a primeira e a última - dessa forma os alunos, que por alguma eventualidade, não participassem de uma aula, conseguiriam acompanhar o resto da turma nas aulas seguintes. Durante 50 
minutos os conceitos seriam apresentados, de maneira teórica e prática, e durante os últimos 30 minutos o espaço seria utilizado para realização de exercícios para fixação do conteúdo, de maneira acompanhada dos ministrantes.

Tabela 1. Relação dos encontros e os temas discutidos

\begin{tabular}{|c|c|}
\hline Encontro & Tema \\
\hline 1 & $\begin{array}{c}\text { Discussão sobre variáveis: o que são, os seus tipos, onde ficam } \\
\text { armazenadas, como declará-las }\end{array}$ \\
\hline 2 & Comandos de Controle I: verdadeiro e falso, condicionais e \\
condicionais aninhados
\end{tabular}

O último encontro foi reservado para a realização de um teste final para avaliar o rendimento dos alunos no decorrer da oficina, com objetivo de verificar o sucesso da ação. Todos os alunos, que alcançaram $70 \%$ de frequência em aulas e $60 \%$ de acertos no teste do último dia, receberam um certificado de conclusão do projeto.

\section{Resultados}

Ao todo, foram recebidas 53 inscrições válidas, porém apenas 24 alunos puderam participar do projeto em decorrência do horário e do campus da UFPel escolhido. Além disso, notou-se que a maior procura pelo projeto foram de alunos pertencentes a cursos da área de exatas - em geral, cursos que possuíam a disciplina de programação de computadores. Porém, a procura pelo projeto, veio de alunos de muitos cursos da UFPel, que pode ser acompanhada na Figura 1.

Todos os alunos que possuiam frequência para realizar o teste final (acima de $75 \%$ ), obtiveram mais que $60 \%$ de acertos, a média geral de nota da turma que participou do teste final foi de 8,6 num total de 10 pontos. Apenas 1 aluno abandonou a oficina. 


\section{Cursos dos Alunos Inscritos}

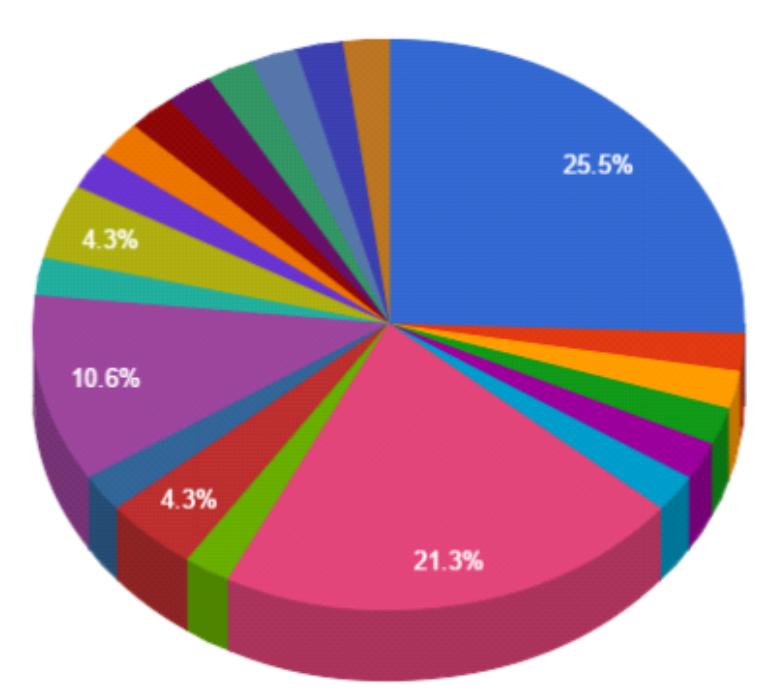

Engenharia Hidrica

Engenharia de Produção

Meteorologia

Geoprocessamento

Engenharia Sanitária e Ambiental

Medicina Veterinária

Engenharia de Controle e Automação

Artes Visuais

Engenharia de Materiais

Jornalismo

Engenharia Eletrônica

Educação Física

Engenharia Geológica

Agronomia

Ciências Econômicas

Engenharia de Produção

Figura 1. Cursos dos alunos que procuraram o projeto de lógica de programação.

\section{Conclusões}

Após a término do curso, notou-se que ele atendeu a proposta, pois todos os encontros ocorreram como planejado. Ratificando o seu sucesso, foi obtido $100 \%$ de aprovação no teste final.

Assim como discutido nos trabalhos correlatos, a ausência de disciplinas de lógica no Ensino Básico possui como consequência a deficiência enfrentada no pensamento crítico e lógico dos alunos. Quando chegam ao curso superior esbarram na dificuldade ocasionada pela carência do raciocínio necessário. É preciso que ações como essa oficina sejam ofertadas, não só para cursos de tecnologia, mas também para escolas de ensino fundamental e para os cursos de graduação diversos, como é o caso deste relato.

Em uma era tecnológica é necessário que a população seja induzida a explorar os recursos computacionais para adaptação e emprego destes em seu cotidiano. $O$ grupo PET-Computação da UFPel percebe esta carência e prevê que nos trabalhos futuros as oficinas sejam realizadas em turnos paralelos, para que mais alunos sejam alcançados, e a extensão também seja contemplada, seja com alunos do ensino básico ou na formação de outros membros da comunidade.

\section{Agradecimentos}

Os autores agradecem o Programa de Educação Tutorial do Ministério da Educação e as agências de fomento CNPq e FAPERGS pelo apoio no desenvolvimento deste trabalho. Ainda, agradecem às coordenações dos cursos de Computação da UFPel pela disponibilização dos recursos para a realização do projeto. 


\section{Referências}

Barbosa, L., S. (2011) “Aprendizado Significativo Aplicado ao Ensino de Algoritmos”. Dissertação (Pós-Graduação em Sistemas de Computação) - Departamento de informática e Matemática Aplicada. Universidade Federal do Rio Grande do Norte, Brasil.

Bosse, Yorah; Gerosa, Marco Aurélio. Reprovações e Trancamentos nas Disciplinas de Introdução à Programação da Universidade de São Paulo: Um Estudo Preliminar. In: WORKSHOP SOBRE EDUCAÇÃO EM COMPUTAÇÃO (WEI), 23., 2015, Recife. Anais: Porto Alegre: Sociedade Brasileira de Computação, 2015. p. 426-435. ISSN 2595-6175.

De Oliveira, Milena; De Souza, Anderson; Ferreira, Aline; Barreiros, Emanoel. Ensino de lógica de programação no ensino fundamental utilizando o Scratch: um relato de experiência. In: WORKSHOP SOBRE EDUCAÇÃO EM COMPUTAÇÃO (WEI), 22., 2014, Brasília. Anais. Porto Alegre: Sociedade Brasileira de Computação, 2014. p. 239-248. ISSN 2595-6175.

França, R. S.; Silva, W. C; Amaral, H. J. C. (2013) "Computino: um jogo destinado à aprendizagem de Números Binários para estudantes da educação básica”. In Anais do XXXIII Congresso da SBC- WEI. Maceió, Brasil.

Francisco, Rodrigo Elias; Pereira Júnior, Cleon X.; Ambrósio, Ana Paula. Grau de Dificuldade de Problemas de Programação Introdutória: Uma Revisão Sistemática da Literatura. In: WORKSHOP SOBRE EDUCAÇÃO EM COMPUTAÇÃO (WEI), 25., 2017, São Paulo. Anais. Porto Alegre: Sociedade Brasileira de Computação, 2017. ISSN 2595-6175.

Mattos, Giorgia de O.; Moreira, Josilene A.; Moura, Ana Flávia S. A.; Nascimento, Andrea B.; Oliveira, Chaenne C. Oficinas de Programação para Meninas: Despertando o Interesse Pela Computação. In: WORKSHOP SOBRE EDUCAÇÃO EM COMPUTAÇÃO (WEI), 26., 2018, Natal. Porto Alegre: Sociedade Brasileira de Computação, 2018. ISSN 2595-6175.

Pereira, Felício Gobbi Hoffmann Santos; De Araújo, Graziela Santos; Cheung, Luciana Montera; De Araujo, Anderson Viçoso; Zunta, Heitor Batistela. Relato da utilização da plataforma App Inventor como ferramenta de ensino de lógica de programação para professores da Rede Básica de Ensino. In: WORKSHOP SOBRE EDUCAÇÃO EM COMPUTAÇÃO (WEI), 28., 2020, Cuiabá. Porto Alegre: Sociedade Brasileira de Computação, 2020. p. 86-90. ISSN 2595-6175.

Schildt, H. C Completo e Total. Brasil: Makron Books, 1996.

Tucker, A. (2006) A Model Curriculum for K-12 Computer Science. Final Report of the ACM K-12 Task Force Curriculum Committee.

Watson, C. and Li, F. W. (2014). Failure rates in introductory programming revisited. In Proceedings of the 2014 conference on Innovation \& technology in computer science Education, pages 39-44. ACM. 